Pacific

Journal of

Mathematics

A VARIATIONAL FORMULA FOR FLOATING BODIES

JOHN MCCUAN 


\title{
A VARIATIONAL FORMULA FOR FLOATING BODIES
}

\author{
JOHN MCCUAN
}

\begin{abstract}
Well known first order necessary conditions for a liquid mass to be in equilibrium in contact with a fixed solid surface declare that the free surface interface has mean curvature prescribed in terms of the bulk accelerations acting on the liquid and meets the solid surface in a materially dependent contact angle. We derive first order necessary conditions for capillary surfaces in equilibrium in contact with solid surfaces which may also be allowed to move. These conditions consist of the same prescribed mean curvature equation for the interface, the same prescribed contact angle condition on the boundary, and an additional integral condition which may be said to involve, somewhat surprisingly, only the wetted region.

An example of the kind of system under consideration is that of a floating ball in a fixed container of liquid. We apply our first order conditions to this particular problem.
\end{abstract}

\section{Introduction: expressions for energy and volume}

In the calculus of variations, one is interested in minimizing an energy functional over some class of admissible functions. The energy is usually assumed to be an integral operator. For example, one often considers

$$
\mathscr{F}[u]=\int_{\Omega} F(x, u, D u) d x+\int_{\partial \Omega} \bar{F}(x, u, D u) d x
$$

where $\Omega$ is a fixed domain in $\mathbb{R}^{n}$ and the competing functions $u$ are all defined on $\bar{\Omega}$.

Following Gauss, a standard approach to the derivation of equations governing equilibrium configurations for liquid masses in contact with solid bounding surfaces is via the calculus of variations. In this context, due to the geometric and in particular parametric nature of the situation, it is more natural to consider energy functionals which are integral operators on a given Riemann surface $M$ and which involve a parametric mapping $X: M \rightarrow \mathbb{R}^{3}$. Though the value of the energy involves

MSC2000: primary 76B45, 76D45, 49K20; secondary 49Q05, 53A10.

Keywords: calculus of variations, capillarity, minimal surfaces, constant mean curvature.

The author is grateful to the Max Planck Institute for Mathematics in the Sciences, which supported this research. 
universally the inclusion of surface area terms for the liquid free surface interface and the wetted region on the solid support surface, the precise identification in the literature of the Riemann surface $M$ and the mapping $X$ is somewhat more obscure.

In most, if not all, cases it is tacitly assumed that the entire configuration is determined by the free surface interface and, hence, that the functional $\mathscr{E}=\mathscr{E}[X]$ has as argument the mapping $X$ which parameterizes the free surface interface. More precisely, it is assumed that the mapping $X$ which determines the free surface interface also determines the contact line (the boundary of the free interface); the contact line determines a particular wetted region on the solid surface; the free surface interface together with the wetted region determine the enclosed volume or liquid mass, and this is enough information to compute the energy. Competing admissible mappings are then naturally considered as those mappings defined on the image $\Lambda$ of $X$, or equivalently on its preimage $M$. Each such free surface interface leads to an admissible configuration defining a liquid mass as just described. From this point of view, the objective may be stated as finding a piecewise $C^{1}$ mapping $X_{0}$ determining a free surface interface for which $\mathscr{E}\left(X_{0}\right) \leq \mathscr{E}(X)$ for all piecewise $C^{1}$ competing or admissible mappings $X$. It should be noted that in this formulation, the energy is given implicitly at least to the extent that a particular integral describing, say, the wetted area, is never written down. Since the first variation formula in this context only involves integrals over $\Lambda$ and $\partial \Lambda$, this point of view seems at first adequately justified, at least formally.

We give here an alternative approach which is somewhat more explicit at least to the extent that the initial energy is given in terms of a well defined integral operator. In situations in which portions of the solid boundary are free to move, or partially free to move, the image of the free surface does not always determine the wetted region or the volume; consider a cylindrical container

$$
\left\{(x, y, z) \in \mathbb{R}^{3}: x^{2}+y^{2}=2,-3 \leq z \leq 1\right\} \cup\left\{(x, y, z) \in \mathbb{R}^{3}: x^{2}+y^{2} \leq 2, z=-3\right\}
$$

with the floating solid cylinder

$$
\left\{(x, y, z) \in \mathbb{R}^{3}: x^{2}+y^{2} \leq 1,-2+h \leq z \leq 1+h\right\}
$$

for $h$ fixed with $-1<h<2$. An admissible interface for every value of $h$ is given by the annulus $\left\{(x, y, 0) \in \mathbb{R}^{3}: 1<x^{2}+y^{2}<2\right\}$; while this interface determines the contact line, the wetted region and enclosed volume both depend explicitly on $h$. In such situations, our alternative and more general approach is more natural, being further justified by the fact that additional integral terms, not expressible as integrals over the free surface interface or its boundary, appear in the first order necessary conditions. Furthermore, the explicit appearance of an independent parameter $h$ in this type of problem suggests an analogous class of 
problems in the nonparametric case different from (1). Namely, one may consider

$$
\mathscr{F}[u, h]=\int_{\Omega} F(x, u, D u, h) d x+\int_{\partial \Omega} \bar{F}(x, u, D u, h) d x,
$$

where the admissible functions $u$ are defined on $\Omega \times \tilde{\Omega}$ for some set $\tilde{\Omega} \in \mathbb{R}^{m}$. We are unaware of any treatment of problems of this sort in the literature, but one can think of some interesting examples with little difficulty.

We now give formal mathematical assumptions used to model capillary systems in somewhat more detail than we have found in the literature. We model solid surfaces by the boundaries of initially prescribed closed sets in $\mathbb{R}^{3}$. The union of all such solid surfaces is denoted by $\Sigma=\partial \mathscr{C}$ where $\mathscr{b}$ denotes the union of the closed sets. We assume, for the moment, that all solid surfaces are fixed, and an interior liquid mass, modeled by an open set $\mathcal{M}$, occupies some portion of $\mathbb{R}^{3} \backslash \mathscr{C}$. The boundary of $\mathcal{M}$ consists of a portion $\mathscr{W}$ in $\Sigma$, which we call the wetted region, and a portion $\Lambda=\partial \mathcal{M} \backslash \mathcal{W}$, the free surface interface.

Given a particular liquid mass $\mathcal{M}_{0}$, we assume $\mathcal{M}_{0}$ and the associated surfaces $\Lambda_{0}$ and $\mathcal{W}_{0}$ admit the structure of abstract Riemannian manifolds (of dimensions 3,2 , and 2 respectively).

Relative to $M_{0}$, we consider the admissible class of liquid mass configurations $\mathcal{M}$ obtained as parameterized images

$$
X: \bar{M}_{0} \rightarrow \mathbb{R}^{3} .
$$

More precisely, we consider liquid masses $M=X\left(M_{0}\right)$ with smooth parameterization $\left.X\right|_{\mathcal{M}_{0}}$; it is assumed that $\partial \mathcal{M}=X\left(\Lambda_{0}\right) \cup X\left(W_{0}\right)$ with free surface interface $\Lambda=X\left(\Lambda_{0}\right)$, parameterized by $\left.X\right|_{\Lambda_{0}}$, and wetted region $\mathcal{W}=X\left(\mathcal{W}_{0}\right)=X\left(\bar{M}_{0}\right) \cap \Sigma$ with parameterization $\left.X\right|_{\mathscr{W}_{0}}$.

Given an admissible liquid mass configuration $\mathcal{M}$, we assume the energy of the system is given by

$$
\mathscr{E}=A(\Lambda)-\beta A(\mathscr{W})
$$

where $A$ denotes the area functional. The constant $\beta$ is called the adhesion coefficient. See [Finn 1986] for further details. For simplicity, we have not included in (2) the possibility that bulk accelerations are acting on the liquid. The applications given in this paper do not involve gravity or other accelerations, but for reference we will briefly indicate below what occurs in this more general setting.

We will assume that each global parameterization, such as $\left.X\right|_{\Lambda_{0}}: \Lambda_{0} \rightarrow \Lambda$, extends smoothly to an open neighborhood of the boundary. In particular, we can parameterize a small neighborhood of every point in the closure of $\Lambda$ locally by some map defined in a neighborhood of $\mathbb{R}^{2}$. We will employ the usual abuse of 


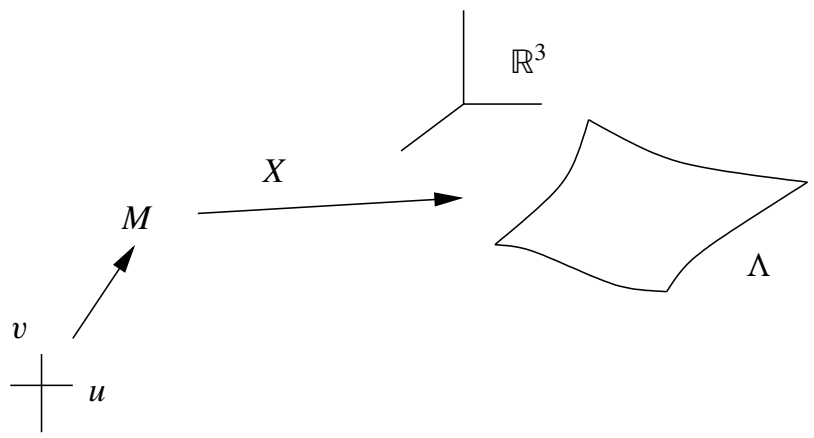

Figure 1. A free surface interface parameterized on an abstract manifold

notation in denoting this local parameterization also by $X$. More generally, we may emphasize the role of either parameter domain $\Lambda_{0}$ or $\mathcal{W}_{0}$ (or their union) as an abstract manifold by denoting it as $M$ and referring to the appropriate restriction of $X: \bar{M}_{0} \rightarrow \mathbb{R}^{3}$ simply as $X: M \rightarrow \mathbb{R}^{3}$. The context is usually adequate to indicate which portion of $\partial \mathcal{M}$ is under consideration. If we wish to emphasize the distinction between the free surface $\Lambda$ and wetted region $W$, we will use a superscript "W" in reference to the wetted region.

Under these assumptions, the area functional can be written explicitly as an integral over a fixed Riemann surface $M$ of the Jacobian scaling factor $\sqrt{\operatorname{det}\left(d X^{T} d X\right)}$ where the superscript " $T$ " denotes the transpose (or adjoint) of the linear transformation; see [Evans and Gariepy 1992]. We can write, for example,

$$
A(\Lambda)=\int_{\Lambda} 1=\int_{\Lambda_{0}} \sqrt{\operatorname{det}\left(d X^{T} d X\right)}=\int_{M} \sqrt{\operatorname{det}\left(d X^{T} d X\right)} .
$$

Using a similar expression for the wetting energy, we see that the energy of admissible configurations is well defined as a functional on pairs of restrictions $X$ : $M=\Lambda_{0} \rightarrow \mathbb{R}^{3}, X^{\mathscr{W}}: M^{\mathscr{W}}=\mathcal{W}_{0} \rightarrow \mathbb{R}^{3}$ on a pair of fixed Riemann surfaces (or alternatively on admissible maps $X$ on the union $M=\Lambda_{0} \cup \mathcal{W}_{0}$ ):

$$
\mathscr{E}(X)=\int_{M} \sqrt{\operatorname{det}\left(d X^{T} d X\right)}-\beta \int_{M^{\mathscr{W}}} \sqrt{\operatorname{det}\left(d X^{\mathscr{W} T} d X^{\mathscr{W}}\right)}
$$

The volume of the liquid mass can also be expressed in terms of integrals over the same parameter domains. In fact, using the divergence theorem

$$
V=\int_{\mathcal{M}} 1=\frac{1}{3} \int_{\mathcal{M}} \operatorname{div}_{\mathbb{R}^{3}} x=\frac{1}{3} \int_{\Lambda} X \cdot N+\frac{1}{3} \int_{\mathscr{W}} X_{0}^{\mathscr{W}} \cdot N_{0}^{\mathscr{W}} .
$$


Thus, we may write

(4) $\left.V(X)=\frac{1}{3} \int_{M} X \cdot N \sqrt{\operatorname{det}\left(d X^{T} d X\right)}+\frac{1}{3} \int_{M^{\mathscr{W}}} X_{0}^{\mathscr{W}} \cdot N_{0}^{\mathscr{W}} \sqrt{\operatorname{det}\left(d X^{\mathscr{W}} T\right.} d X^{\mathscr{W}}\right)$.

Expressions (3) and (4) provide the basis for the calculations in the next section.

When bulk accelerations are considered, one encounters in the energy an additional term of the form

$$
\mathscr{G}=\int_{\mathcal{M}} g
$$

where $g$ is a given scalar function of position. I am unaware of a means to express this term as an integral over $\partial M_{0}$. Thus, we are apparently forced to use the full information of the mapping $X: \bar{M}_{0} \rightarrow \mathbb{R}^{3}$ associated with an admissible variation of the bulk liquid and admit the dependence of the energy on the third restriction $\left.X\right|_{\mu_{0}}: M_{0} \rightarrow \mathbb{R}^{3}$, which we also denote by $X:$

$$
\mathscr{G}(X)=\int_{\mathcal{M}_{0}} g \circ X|\operatorname{det} D X|
$$

\section{General formulae}

Given a liquid mass configuration as described above, we wish to consider a variation $X: \bar{M}_{0} \times(-\epsilon, \epsilon) \rightarrow \mathbb{R}^{3}$ such that $X(\cdot ; 0)=\operatorname{id}_{\bar{M}_{0}}(\cdot)$ and for each fixed $t \in(-\epsilon, \epsilon)$, the two restrictions

$$
\left.X\right|_{\Lambda_{0} \times\{t\}}: \Lambda_{0}=M \rightarrow \mathbb{R}^{3} \text { and }\left.X\right|_{\mathcal{W}_{0} \times\{t\}}: \mathcal{W}_{0}=M^{\mathscr{W}} \rightarrow \mathbb{R}^{3}
$$

which parameterize images $\Lambda$ and $\mathcal{W}$ respectively, arise as restrictions to $\Lambda_{0}$ and $W_{0}$ of the global map $X: \bar{M}_{0} \rightarrow \mathbb{R}^{3}$ associated to an admissible liquid mass $M=$ $M_{t}$. The variations under consideration here are required to be smooth so that the functions

$$
\mathscr{E}(t)=A(\Lambda)-\beta A(\mathscr{W}) \text { and } V(t)=\frac{1}{3} \int_{\Lambda} X \cdot N+\frac{1}{3} \int_{\mathscr{W}} X^{\mathscr{W}} \cdot N^{\mathscr{W}}
$$

are differentiable on $(-\epsilon, \epsilon)$.

Finally, we note the compatibility of the restrictions to $W_{0}$ and $\Lambda_{0}$ along their common boundary:

$$
X^{\mathscr{W}}(q ; t)=X(q ; t) \text { for every } q \in \partial M^{\mathscr{W}}=\partial M
$$

This simple relation will play a key role in our derivation of a variational formula for floating bodies.

With no bulk accelerations, no condition on the interior restriction $\left.X\right|_{\mu_{0} \times\{t\}}$ need be considered, and this part of the global map can be ignored. 

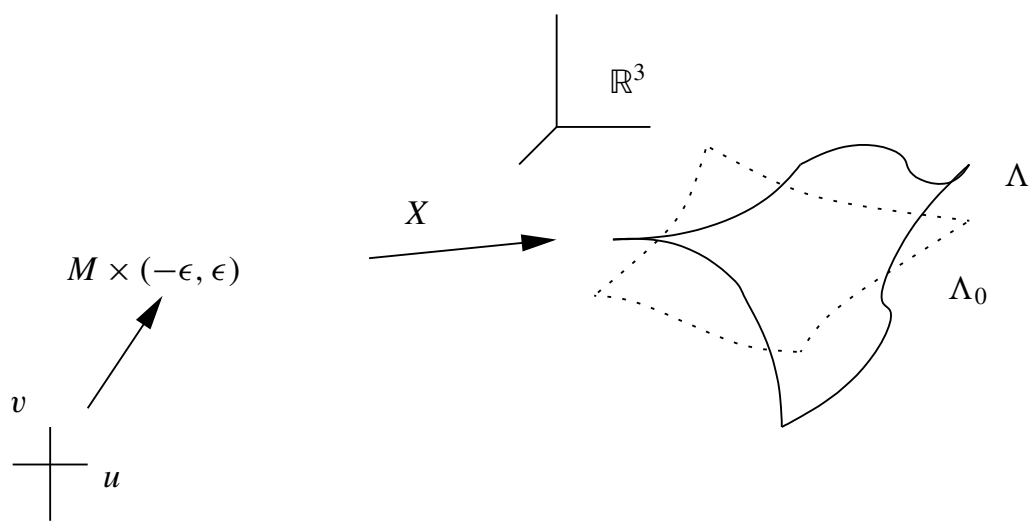

Figure 2. Variation of $\Lambda_{0}$.

We wish to compute $\dot{\mathscr{E}}=d \mathscr{E} / d t$. We begin with $A(\Lambda)$.

$$
A(\Lambda)=\int_{\Lambda} 1=\sum_{U} \int_{U} \sqrt{E G-F^{2}}
$$

where we have written the abstract manifold $M$ (up to a set of measure zero) as a disjoint union of images of coordinate neighborhoods $U$ in the $u, v$-plane and $E$, $F$, and $G$ are the coefficients of the first fundamental form on a given neighborhood $U$. That is, taking $X$ to be locally defined on $U$, we have

$$
E=\left|X_{u}\right|^{2}, \quad F=X_{u} \cdot X_{v}, \quad G=\left|X_{v}\right|^{2}, \quad \sqrt{E G-F^{2}}=\left|X_{u} \times X_{v}\right| .
$$

Thus we obtain

$$
\begin{aligned}
\frac{d}{d t} \int_{U} \sqrt{E G-F^{2}} & =\int_{U} \frac{\left(X_{u} \times X_{v}\right) \cdot\left(\dot{X}_{u} \times X_{v}+X_{u} \times \dot{X}_{v}\right)}{\sqrt{E G-F^{2}}} \\
& =\int_{U} \frac{\left(X_{u} \cdot \dot{X}_{u}\right) G-\left(X_{v} \cdot \dot{X}_{u}\right) F+\left(X_{v} \cdot \dot{X}_{v}\right) E-\left(X_{u} \cdot \dot{X}_{v}\right) F}{\sqrt{E G-F^{2}}}
\end{aligned}
$$

Now, if we write $\dot{X}=\phi N+\psi X_{u}+\eta X_{v}=\phi N+\dot{X}^{\mathrm{T}}$, where $N$ is a unit normal to $\Lambda$, then

$$
\begin{aligned}
& \dot{X}_{u}=\phi_{u} N+\phi N_{u}+\psi_{u} X_{u}+\psi X_{u u}+\eta_{u} X_{v}+\eta X_{u v}, \\
& \dot{X}_{v}=\phi_{v} N+\phi N_{v}+\psi_{v} X_{u}+\psi X_{u v}+\eta_{v} X_{v}+\eta X_{v v},
\end{aligned}
$$


so that

$$
\begin{aligned}
& X_{u} \cdot \dot{X}_{u}=-\phi e+\psi_{u} E+\frac{1}{2} \psi E_{u}+\eta_{u} F+\frac{1}{2} \eta E_{v}, \\
& X_{v} \cdot \dot{X}_{u}=-\phi f+\psi_{u} F+\psi F_{u}-\frac{1}{2} \psi E_{v}+\eta_{u} G+\frac{1}{2} \eta G_{u}, \\
& X_{u} \cdot \dot{X}_{v}=-\phi f+\psi_{v} E+\frac{1}{2} \psi E_{v}+\eta_{v} F+\eta F_{v}-\frac{1}{2} \eta G_{u}, \\
& X_{v} \cdot \dot{X}_{v}=-\phi g+\psi_{v} F+\frac{1}{2} \psi G_{u}+\eta_{v} G+\frac{1}{2} \eta G_{v},
\end{aligned}
$$

where $e=X_{u u} \cdot N, f=X_{u v} \cdot N$, and $g=X_{v v} \cdot N$ are the coefficients of the second fundamental form. Multiplying the expressions above by the appropriate factors, adding them together and grouping like terms we obtain

$$
\begin{aligned}
\left(X_{u} \cdot \dot{X}_{u}\right) G-\left(X_{v} \cdot \dot{X}_{u}\right) F+\left(X_{v} \cdot \dot{X}_{v}\right) E-\left(X_{u} \cdot \dot{X}_{v}\right) F & \\
=-\phi(e G-2 f F+g E)+\psi_{u}(E G & \left.-F^{2}\right)+\frac{1}{2} \psi\left(E G-F^{2}\right)_{u} \\
& +\eta_{v}\left(E G-F^{2}\right)+\frac{1}{2} \eta\left(E G-F^{2}\right)_{v} .
\end{aligned}
$$

Thus, we have

$$
\begin{aligned}
& \frac{d}{d t} \int_{U} \sqrt{E G-F^{2}} \\
& =-\int_{U} \phi \frac{e G-2 f F+g E}{\sqrt{E G-F^{2}}}+\int_{U} \psi_{u} \sqrt{E G-F^{2}}+\psi \frac{\left(E G-F^{2}\right)_{u}}{2 \sqrt{E G-F^{2}}} \\
& \quad+\int_{U} \eta_{v} \sqrt{E G-F^{2}}+\eta \frac{\left(E G-F^{2}\right)_{v}}{2 \sqrt{E G-F^{2}}} \\
& =-\int_{U} \phi \frac{e G-2 f F+g E}{\sqrt{E G-F^{2}}}+\int_{U}\left(\psi \sqrt{E G-F^{2}}\right)_{u}+\left(\eta \sqrt{E G-F^{2}}\right)_{v} .
\end{aligned}
$$

Finally, summing over the neighborhoods $U$ we have

$$
\begin{aligned}
\dot{A}(\Lambda) & =-\int_{\Lambda} 2 H \phi+\sum_{U} \int_{U} \frac{\left(\psi \sqrt{E G-F^{2}}\right)_{u}+\left(\eta \sqrt{E G-F^{2}}\right)_{v}}{\sqrt{E G-F^{2}}} \sqrt{E G-F^{2}} \\
& =-\int_{\Lambda} 2 H \phi+\int_{\Lambda} \operatorname{div}_{\Lambda} \dot{X}^{\mathrm{T}} \\
& =-\int_{\Lambda} 2 H X \cdot N+\int_{\partial \Lambda} \dot{X}^{\mathrm{T}} \cdot \vec{n},
\end{aligned}
$$

where $H$ is the mean curvature of $\Lambda, \vec{n}$ is the outward conormal to $\partial \Lambda$ and the last term comes from the divergence theorem. We note finally, that $\dot{X}^{\mathrm{T}} \cdot \vec{n}=\dot{X} \cdot \vec{n}$ because, $N \cdot \vec{n}=0$.

This formula should be recognized as the general formula for the first variation of area for an arbitrary surface in $\mathbb{R}^{3}$ under an arbitrary vector-valued variation. While the derivation above is somewhat more general than that of [Wente 1966] or [Finn 1986], since we allow arbitrary variations, Spivak [1979] derives an even 
more general version which allows arbitrary dimension and also immersion in an arbitrary submanifold. We felt it was worthwhile, however, to derive the generalization in the limited context of the present discussion.

We may apply the same calculation to the variation of $\mathcal{W}$ by $X^{\mathscr{W}}$ and obtain

$$
\dot{A}(\mathscr{W})=\int_{\partial \Lambda} \dot{X} \cdot \vec{v}
$$

where $v$ is the outward conormal to $\partial W=\partial \Lambda$ with respect to $W$. Notice from (5) that

$$
\dot{X}^{W}(q ; t)=\dot{X}(q ; t)
$$

for every $q \in \partial M^{\mathscr{W}}$, and in the case of a fixed rigid bounding surface $\Sigma$, we are requiring $\dot{X}^{W}=\dot{X}$ to be tangent to $\Sigma$ along $\partial \Lambda$. This is why the surface integral of $2 H^{W} \dot{X} \cdot N^{W}$ does not appear in (6). Moreover, along $\partial \Lambda$ we may write $\dot{X}=$ $(\dot{X} \cdot \vec{v}) \vec{v}+\delta \vec{t}$ where $\vec{t}$ is a unit tangent to $\partial \Lambda$, so that

$$
\dot{\mathscr{E}}=-\int_{\Lambda} 2 H X \cdot N+\int_{\partial \Lambda} \dot{X} \cdot \vec{v}(\vec{v} \cdot \vec{n}-\beta) .
$$

If $\gamma$ is the angle measured inside $\mathcal{M}$ between $\Lambda$ and $\Sigma$ along $\partial \Lambda$, then we obtain the familiar form

$$
\dot{\mathscr{E}}=-\int_{\Lambda} 2 H X \cdot N+\int_{\partial \Lambda}(\cos \gamma-\beta) \dot{X} \cdot \vec{v} .
$$

We next proceed to derive an expression for

$$
\dot{V}=\frac{d}{d t} V(t)
$$

where

$$
V=V(t)=\int_{\mathcal{M}} 1=\frac{1}{3} \int_{\mathcal{M}} \operatorname{div}_{\mathbb{R}^{3}} x=\frac{1}{3} \int_{\Lambda} X \cdot N+\frac{1}{3} \int_{\mathscr{W}} X^{\mathscr{W}} \cdot N^{W}
$$

by the divergence theorem.

Note. The expression used for the change in $V(\mathcal{M})$ in [Barbosa et al. 1988] and referenced by [Ros and Souam 1997] apparently assumes that the variation mapping $X$ (on $M \times[0, \epsilon]$ ) gives a parameterization of the volume under consideration. By following Wente [1966] (who followed Blaschke [1930]) we get an expression which is valid under this assumption and also without it. The computation, however, is a little more involved.

It is interesting to note that earlier, Barbosa and do Carmo [1984] use also Blaschke's expression for the volume. Presumably, the formulation in their latter paper [1988] is a consequence of working in a curved ambient manifold. This suggests the following question: Is there a generalization of the trivial formula $\operatorname{div}_{\mathbb{R}^{n}} x=n$ to situations in which $\mathbb{R}^{n}$ is replaced by an $n$-dimensional manifold? 
The question in one lower dimension, also has relevance for the main problem under consideration in this paper, because if this were the case, it might be possible to replace the wetting energy term with an integral around $\partial \Lambda$ and, hence, simplify the overall construction. It should be noted, of course that in the case of a floating object, that vector field would be expected to depend on the position of the floating object.

Finally, in using Blaschke's expression for the volume, we have effectively replaced an integral over $\mathcal{M}$ by one over $\partial \mathcal{M}$. If we are willing to consider variation of the entire liquid mass (which we are apparently forced to do below to accommodate bulk accelerations), then an alternative approach is possible. This approach is presented in the context of the more complicated situation below.

Returning to the variation of Blaschke's volume expression, we begin as before, writing $\Lambda$ as a union of parameter neighborhoods $X(U)$. Then

$$
\frac{d}{d t} \int_{\Lambda} X \cdot N=\sum_{U} \frac{d}{d t} \int_{U} X \cdot\left(X_{u} \times X_{v}\right) .
$$

For each local domain $U$,

$$
\frac{d}{d t} \int_{U} X \cdot\left(X_{u} \times X_{v}\right)=\int_{U} \dot{X} \cdot\left(X_{u} \times X_{v}\right)+X \cdot\left(\dot{X}_{u} \times X_{v}\right)+X \cdot\left(X_{u} \times \dot{X}_{v}\right) .
$$

Considering the last two terms in this expression,

$$
\begin{aligned}
X \cdot\left(\dot{X}_{u} \times X_{v}\right)+X \cdot\left(X_{u} \times \dot{X}_{v}\right) & \\
= & {\left[X \cdot\left(\dot{X} \times X_{v}\right)\right]_{u}+\left[X \cdot\left(X_{u} \times \dot{X}\right)\right]_{v}-X_{u} \cdot\left(\dot{X} \times X_{v}\right)-X \cdot\left(X_{u v} \times \dot{X}\right) } \\
& \quad-X_{v} \cdot\left(X_{u} \times \dot{X}\right)-X \cdot\left(\dot{X} \times X_{u v}\right) \\
= & {\left[X \cdot\left(\dot{X} \times X_{v}\right)\right]_{u}+\left[X \cdot\left(X_{u} \times \dot{X}\right)\right]_{v}+2 \dot{X} \cdot\left(X_{u} \times X_{v}\right) . }
\end{aligned}
$$

In the last line, we have used the identity $-X_{v} \cdot\left(X_{u} \times \dot{X}\right)=\operatorname{vol}\left(X_{u}, X_{v}, \dot{X}\right)=$ $-X_{u} \cdot\left(\dot{X} \times X_{v}\right)$ where $\operatorname{vol}\left(X_{u}, X_{v}, \dot{X}\right)=\dot{X} \cdot\left(X_{u} \times X_{v}\right)$ is the volume of the parallelepiped spanned by $\dot{X}, X_{u}$ and $X_{v}$ (up to a sign). Substituting this into the integral under consideration, we find

$$
\frac{d}{d t} \int_{U} X \cdot\left(X_{u} \times X_{v}\right)=3 \int_{U} \dot{X} \cdot\left(X_{u} \times X_{v}\right)+\int_{U}\left[X \cdot\left(\dot{X} \times X_{v}\right)\right]_{u}+\left[X \cdot\left(X_{u} \times \dot{X}\right)\right]_{v} .
$$

We wish to express the last integrand as the local expression of the divergence of a globally defined vector field on $\Lambda$. Substituting the expression

$$
\dot{X}=\phi N+\psi X_{u}+\eta X_{v}=\phi N+\dot{X}^{\mathrm{T}}
$$


for $\dot{X}$, we find that the integrand can be written as

$$
\begin{aligned}
{\left[X \cdot\left(\dot{X} \times X_{v}\right)\right]_{u}+\left[X \cdot\left(X_{u} \times \dot{X}\right)\right]_{v} } & =\left[X \cdot \frac{\phi\left(X_{u} \times X_{v}\right) \times X_{v}}{\sqrt{E G-F^{2}}}\right]_{u}+\left[\sqrt{E G-F^{2}} \psi X \cdot N\right]_{u} \\
& +\left[X \cdot \frac{\phi X_{u} \times\left(X_{u} \times X_{v}\right)}{\sqrt{E G-F^{2}}}\right]_{v}+\left[\sqrt{E G-F^{2}} \eta X \cdot N\right]_{v} .
\end{aligned}
$$

The second and fourth terms sum to precisely

$$
\sqrt{E G-F^{2}} \operatorname{div}_{\Lambda}\left[(X \cdot N) \dot{X}^{\mathrm{T}}\right] .
$$

Applying the triple cross product rule, we find

$$
\left(X_{u} \times X_{v}\right) \times X_{v}=-G X_{u}+F X_{v} \text { and } X_{u} \times\left(X_{u} \times X_{v}\right)=F X_{u}-E X_{v} \text {. }
$$

Thus, the first and third terms sum to

$$
\begin{aligned}
& {\left[\sqrt{E G-F^{2}} \phi \frac{-G X \cdot X_{u}+F X \cdot X_{v}}{E G-F^{2}}\right]_{u}} \\
& +\left[\sqrt{E G-F^{2}} \phi \frac{-F X \cdot X_{u}-E X \cdot X_{v}}{E G-F^{2}}\right]_{v} .
\end{aligned}
$$

Comparing this with the expression of a general vector field in local coordinates, we see that this is

$$
-\sqrt{E G-F^{2}} \operatorname{div}_{\Lambda}\left[\phi X^{\mathrm{T}}\right] .
$$

Going back to the original integral, we have

$$
\frac{d}{d t} \int_{U} X \cdot\left(X_{u} \times X_{v}\right)=3 \int_{X(U)} \dot{X} \cdot N+\int_{X(U)} \operatorname{div}_{\Lambda}\left[\phi \dot{X}^{\mathrm{T}}-(X \cdot N) X^{\mathrm{T}}\right] .
$$

Properly speaking, the expressions $X$ and $\dot{X}$ in the integrals on the right side of this equation represent respectively $X\left(X^{-1}(x ; t) ; t\right)$ and $\dot{X}\left(X^{-1}(x ; t) ; t\right)$ for $x \in$ $X(U) \subset \Lambda$. The function $v(x ; t):=\dot{X}\left(X^{-1}(\cdot ; t) ; t\right)$ will play an important role in the case of bulk accelerations below.

Finally summing over all neighborhoods $U$ and recalling that $\phi=\dot{X} \cdot N$, we have

$$
\begin{aligned}
\frac{1}{3} \frac{d}{d t} \int_{\Lambda} X \cdot N & =\int_{\Lambda} \dot{X} \cdot N+\frac{1}{3} \int_{\Lambda} \operatorname{div}_{\Lambda}\left[(X \cdot N) \dot{X}^{\mathrm{T}}-(\dot{X} \cdot N) X^{\mathrm{T}}\right] \\
& =\int_{\Lambda} \dot{X} \cdot N+\frac{1}{3} \int_{\partial \Lambda}\left[(X \cdot N) \dot{X}^{\mathrm{T}}-(\dot{X} \cdot N) X^{\mathrm{T}}\right] \cdot \vec{n} \\
& =\int_{\Lambda} \dot{X} \cdot N+\frac{1}{3} \int_{\partial \Lambda}[(X \cdot N) \dot{X}-(\dot{X} \cdot N) X] \cdot \vec{n} .
\end{aligned}
$$

Again, we have used the divergence theorem. The last line results from the fact that the vectors $(X \cdot N) \dot{X}$ and $(\dot{X} \cdot N) X$ have the same component in the direction $N$. 
The same calculation applies to the integral over $\mathcal{W}$ appearing in the expression for $V$ so that

where

$$
\dot{V}=\int_{\Lambda} \dot{X} \cdot N+\int_{\mathscr{W}} \dot{X}^{\mathscr{W}} \cdot N^{\mathscr{W}}+J
$$

$$
3 J=\int_{\partial \Lambda}[(X \cdot N) \dot{X}-(\dot{X} \cdot N) X] \cdot \vec{n}+\left[\left(X \cdot N^{W}\right) \dot{X}-\left(\dot{X} \cdot N^{W}\right) X\right] \cdot \vec{v} .
$$

It would be usual at this point to assume $\dot{X} \cdot N^{\text {W }}=0$ along $\partial \Lambda$ so that one of the boundary integral terms vanishes. In keeping with the use of quite general vectorvalued variations, we will avoid such an assumption. We will substitute rather

$$
\dot{X}=\phi N+\delta \vec{n}+\eta \vec{t}, \quad \vec{v}=(\vec{v} \cdot N) N+(\vec{v} \cdot \vec{n}) \vec{n}, \quad N^{\mathscr{W}}=-(\vec{v} \cdot \vec{n}) N+(\vec{v} \cdot N) \vec{n},
$$

where $\vec{t}$ is a vector tangent to $\partial \Lambda$. In this way, we find

$$
\begin{aligned}
& {[(X \cdot N) \dot{X}-(\dot{X} \cdot N) X] \cdot \vec{n}+\left[\left(X \cdot N^{\mathscr{W}}\right) \dot{X}-\left(\dot{X} \cdot N^{\mathscr{W}}\right) X\right] \cdot \vec{v}} \\
& =\phi\left[-X \cdot \vec{n}+\left(X \cdot N^{W}\right)(\vec{v} \cdot N)+(\vec{v} \cdot \vec{n})(X \cdot \vec{v})\right] \\
& \quad \quad+\delta\left[X \cdot N+\left(X \cdot N^{\mathscr{W}}\right)(\vec{v} \cdot \vec{n})-(\vec{v} \cdot N)(X \cdot \vec{v})\right] \\
& =\phi\left[-(X \cdot \vec{n})\left(1-(\vec{v} \cdot \vec{n})^{2}-(\vec{v} \cdot N)^{2}\right)\right]+\delta\left[(X \cdot N)\left(1-(\vec{v} \cdot \vec{n})^{2}-(\vec{v} \cdot N)^{2}\right)\right] \\
& =0 .
\end{aligned}
$$

Thus, we have a general variational formula for volume with respect to an arbitrary admissible vector-valued variation:

$$
\dot{V}=\int_{\Lambda} \dot{X} \cdot N+\int_{\mathscr{W}} \dot{X}^{\mathscr{W}} \cdot N^{\mathscr{W}}
$$

In the situation presently under consideration, we also have that $\dot{X}^{W}$ is in the tangent space of $\Sigma$, so that the second integral also vanishes, and we obtain the familiar

$$
\dot{V}=\int_{\Lambda} \dot{X} \cdot N
$$

If the additional term $G_{\mathcal{M}}=\int_{\mathcal{M}} g$ is included in the energy, we require in addition that the third restriction of our variation $\left.X\right|_{\mu_{0} \times\{t\}}: \mu_{0} \rightarrow \mathbb{R}^{3}$ parameterizes its image which is required to be the admissible liquid mass $M=M_{t}$ bounded by $\Lambda$ and $\mathcal{W}$. In this more complicated situation, we first write

$$
\varphi=\int_{M_{0}} g \circ X \operatorname{det} D X,
$$

where we have omitted the absolute values on the Jacobian factor for small $t$ under the assumption of smoothness in $t$ on the third restriction and the observation that $\operatorname{det} D X(\cdot ; 0)=1>0$. 
This puts us in a position to apply Euler's first kinematical theorem - identity (4.1) in [Serrin 1959] — to obtain

$$
\dot{\varphi}=\int_{\mathcal{M}}\left[D g \cdot v+g \operatorname{div}_{\mathbb{R}^{3}} v\right]=\int_{\mathcal{M}} \operatorname{div}_{\mathbb{R}^{3}}[g v]=\int_{\Lambda} g \dot{X} \cdot N+\int_{\mathscr{W}} g \dot{X}^{W} \cdot N^{W}
$$

by the divergence theorem, where $v(x ; t)=\dot{X}\left(X^{-1}(x ; t) ; t\right)$ is the spatial velocity of the flow induced by the variation. (In the last expression, we have returned to the use of $\dot{X}$ to represent $v(\cdot ; t)$.) For a detailed derivation of the kinematical identity in the special case under consideration, see the appendix to [McCuan 2006].

If $\Sigma$ is a fixed rigid surface, $\dot{X}^{W} \cdot N^{\mathscr{W}}=0$ as before, and we obtain, in complete generality, the formula

$$
\dot{\varphi}=\int_{\Lambda} g \dot{X} \cdot N
$$

which was obtained in special cases in [Wente 1966] and [Finn 1986] but not considered in [Ros and Souam 1997] or [Spivak 1979].

Note: If one is willing to admit the full variation of the interior liquid mass, this approach gives also, in the special case $g \equiv 1$, formulae (8) and (9).

The next step in developing the variational theory of these surfaces would be to introduce a Lagrange parameter corresponding to the volume constraint of the problem. We will have the opportunity to consider this topic under more general circumstances below, and hence move directly to those considerations.

\section{Movable boundaries}

We now wish to allow portions of the rigid bounding surface $\Sigma$ to assume the character of free floating or partially free floating rigid obstacles. To this end, we assume $\Sigma_{0}$ (and correspondingly the relevant abstract manifold $M^{\mathscr{W}}=W_{0}$ ) to be composed of disjoint components $\Sigma_{s}$ and $\Sigma_{m}$. The former portions remain fixed, or stationary, as in the discussion above, while the latter are movable in the sense we now describe.

Let $w=w(x ; h)$ be a family of rigid motions of $\mathbb{R}^{3}$ indexed by and smoothly depending on the parameter $h$ which for simplicity we will assume is real-valued. Correspondingly, we will generalize our notion of variation to that of smooth maps $X: \bar{M}_{0} \times(-\delta, \delta) \times(-\epsilon, \epsilon) \rightarrow \mathbb{R}^{3}$, which for each fixed $h \in(-\delta, \delta)$ satisfy the conditions set forth in the previous section, with one slight modification. The new parameter $h$ lying between $-\delta$ and $\delta$ will index the position of the movable portion of $\Sigma$, so that for fixed $h$ the image of $X_{m}^{W}$ is required to lie in $w\left(\Sigma_{m} ; h\right)$. Otherwise, the parameter $t$ on $(-\epsilon, \epsilon)$ is the variation parameter as before. When convenient, we will continue to refer to the union $M_{s}^{\mathscr{W}} \cup M_{m}^{\mathscr{W}}=\mathscr{W}_{0 s} \cup \mathcal{W}_{0 m}$ simply as $M^{\mathscr{W}}$. As we have denoted derivatives with respect to $t$ with the customary dot above the letter, we will denote derivatives with respect to $h$ with an acute accent:' . For each 
$h$ and $t$, the energy of and volume associated with the configuration are given by the same expressions as before.

We will assume that $\mathscr{E}\left(X_{0}, X_{0}^{\mathscr{W}}\right) \leq \mathscr{E}\left(X, X^{\mathscr{W}}\right)$ for all $C^{1}$ admissible variations $\left(X, X^{\mathscr{W}}\right)$ in a $C^{0}$ neighborhood of $\left(X_{0}, X_{0}^{\mathscr{W}}\right)$ whose associated volume is also constrained to match that of the original minimizing configuration.

We give now a brief justification for the existence of a Lagrange multiplier in this problem. The argument is slightly more general than that seen for integral operators of the form (1) because we avoid the use of "additive variations," i.e., the usual $u+\epsilon \phi$. This is essentially an avoidance of assuming a linear structure in the class of admissible variations. The argument is also somewhat different than that usually given for Fréchet differentiable functionals as in [Gelfand and Fomin 1963] or for Gâteaux differentiable functionals as in [Sagan 1969] or [Ekeland and Temam 1976] for essentially the same reason.

We begin with a standard single parameter variation $Y: \bar{M}_{0} \times(-\epsilon, \epsilon) \rightarrow \mathbb{R}^{3}$ of $\mu_{0}$ for which the volume has nonvanishing first variation. Examination of the variational formula for volume indicates that this is always possible; we can arrange for example that the first restriction (to $\Lambda_{0}$ ) satisfies $\dot{Y} \cdot N \geq 0$ with strict inequality on some open subset of $\Lambda_{0}$. We denote the parameter for this variation by $\tau$ so that $Y=Y(p ; \tau)$. Corresponding to each fixed $\tau$, we consider a further variation $X=X(p ; h ; t ; \tau)$ with $X(p ; 0 ; 0 ; \tau)=Y(p ; \tau)$. This construction results in two smooth real-valued functions of three variables,

$$
f(h, t, \tau)=\mathscr{E}\left(X, X^{\mathscr{W}}\right) \quad \text { and } \quad g(h, t, \tau)=V\left(X, X^{\mathscr{W}}\right),
$$

having the property that $f(0,0,0)$ is a minimum for $f(h, t, \tau)$ subject to the constraint $g(h, t, \tau)=V\left(X_{0}, X_{0}^{\mathscr{W}}\right)$. The usual principle of Lagrange applied to these functions asserts the gradients of $f$ and $g$ must be parallel at the origin; that is,

$$
D f(0,0,0)=\lambda D g(0,0,0)
$$

for some constant $\lambda$. This results in three equations, the first two of which are

$$
\mathscr{\mathscr { E }}-\lambda \dot{V}=0 \quad \text { and } \quad \dot{\mathscr{E}}-\lambda \dot{V}=0,
$$

where everything is evaluated at $h=t=0$. We have computed expressions (7) and (9) for $\dot{\mathscr{E}}$ and $\dot{V}$ above, so that the second equation becomes

$$
\dot{\mathscr{E}}-\lambda \dot{V}=-\int_{\Lambda_{0}}(2 H+\lambda) \dot{X} \cdot N+\int_{\partial \Lambda_{0}}(\cos \gamma-\beta) \dot{X} \cdot \vec{n}=0 .
$$

By taking first interior variations (for which $\dot{X} \equiv 0$ on $\partial \Lambda_{0}$ ) we find

$$
2 H=-\lambda=\text { constant } \text { on } \Lambda_{0} .
$$


Thus, the first integral vanishes, and we can take $\dot{X} \cdot \vec{n}$ arbitrary on $\partial \Lambda_{0}$, so that

$$
\cos \gamma=\beta=\text { constant } \quad \text { on } \partial \Lambda_{0} .
$$

These are the usual first order necessary conditions for a capillary surface in zero gravity. The inclusion of $\varphi$ evidently leads also to the usual prescribed mean curvature equation $2 H=g-\lambda$ where $g$ is a volumetric potential energy density appropriate to any accelerations present.

We now turn to the first equation in (10). Applying the reasoning that led to $\dot{\mathscr{E}}$ but differentiating instead with respect to $h$, we note that there is no reason to believe $\bar{X}^{W}$ lies in the tangent space to $\Sigma$. Thus, we obtain a variational expression with all terms present:

$$
\begin{array}{r}
\mathscr{E}-\lambda \dot{V}=-\int_{\Lambda} 2 H \dot{X} \cdot N+\int_{\partial \Lambda} \dot{X} \cdot \vec{n}+\beta \int_{\mathscr{W}} 2 H^{W} \dot{X}^{W} \cdot N^{\mathscr{W}}-\beta \int_{\partial \Lambda} \dot{X} \cdot \vec{v} \\
-\lambda \int_{\Lambda} \dot{X} \cdot N-\lambda \int_{\mathscr{W}} \dot{X}^{W} \cdot N^{W},
\end{array}
$$

where $H^{\mathscr{W}}$ is the mean curvature on the wetted region $\mathcal{W}$. We note, that we could, of course eliminate some of the integrals over portions $\mathcal{W}_{s}$ of $\mathcal{W}$ which are stationary, but we will save on subscripts by including them for the moment. We can use the prescribed mean curvature equation to cancel the integrals over $\Lambda$, and we may also substitute the contact angle boundary condition so that for $\Lambda=\Lambda_{0}$ and $\Sigma=\Sigma_{0}$ we have

$$
\int_{\partial \Lambda} \dot{X} \cdot(\vec{n}-\cos \gamma \vec{v})+\cos \gamma \int_{\mathscr{W}} 2 H^{\mathscr{W}} \dot{X}^{\mathscr{W}} \cdot N^{\mathscr{W}}+\int_{\mathscr{W}} 2 H \dot{X}^{\mathscr{W}} \cdot N^{\mathscr{W}}=0 .
$$

We have moved the constant $2 H$ inside the integral; it may be easily checked that this same formula holds in the case of bulk accelerations where $2 H=g-\lambda$ may be nonconstant. Noting that $\vec{n}-\cos \gamma \vec{v}=\vec{n}-(\vec{n} \cdot \vec{v}) \vec{v}$ and that $N^{\mathscr{W}}$ and $\vec{v}$ form an orthonormal basis, we see that $\vec{n}-\cos \gamma \vec{v}=\sin \gamma N^{\mathscr{W}}$. Thus the condition becomes

$$
\int_{\partial \Lambda} \sin \gamma \dot{X} \cdot N^{\mathscr{W}}+\cos \gamma \int_{\mathscr{W}} 2 H^{\mathscr{W}} \dot{X}^{\mathscr{W}} \cdot N^{\mathscr{W}}+\int_{\mathscr{W}} 2 H \dot{X}^{\mathscr{W}} \cdot N^{\mathscr{W}}=0 .
$$

This is a first version of our new first order condition which applies to situations in which the solid support structures are deformable. We have not used, however, any special properties of the deformation $X^{W}$ and of the smooth family of rigid motions $w: \mathbb{R}^{3} \times(-\delta, \delta) \rightarrow \mathbb{R}^{3}$. Using these properties should allow us to express the formula in a manner that is essentially independent of $\dot{X}^{\mathscr{W}}$ in the interior of $\mathscr{W}$. The crucial observation is that because

$$
\Sigma_{m ; h}=\Sigma_{h}=w\left(\Sigma_{m} ; h\right)
$$


we have

$$
w^{-1}\left(X^{\mathscr{W}}\right) \in \Sigma_{0}
$$

where we have suppressed the subscript " $m$," and $X^{\mathscr{W}}$ denotes $X_{m}^{\mathscr{W}}(x ; h, t) ; h$. (We note that in the following discussion, we will also consistently suppress $t$ dependence.)

The inclusion (12) implies

$$
D w^{-1}\left(X^{\mathscr{W}}\right) \dot{X}^{\mathscr{W}}+\dot{w}^{-1}\left(X^{\mathscr{W}}\right) \in T_{w^{-1}\left(X^{\mathscr{W}}\right)} \Sigma_{0} .
$$

Recall, however, that for fixed $h$, the transformations $w$ and $w^{-1}$ are rigid motions. Therefore, we know that, for example, $w-w(0)$ is linear and has a corresponding matrix $D w$ which depends only on $h$. Since multiplication by this matrix also corresponds to the differential mapping $d w: T_{w^{-1}\left(X^{W}\right)} \Sigma_{0} \rightarrow T_{X^{W}} \Sigma$ of tangent spaces where $\Sigma=\Sigma_{h}$, we find that

$$
\dot{X}^{W}+D w \dot{w}^{-1}\left(X^{\mathscr{W}}\right) \in T_{X^{W}} \Sigma .
$$

Differentiating the defining relation $w\left(w^{-1}(x ; h) ; h\right)=x$ with respect to $h$, we find

$$
D w\left(w^{-1}(x ; h) ; h\right) \dot{w}^{-1}(x ; h)=-w\left(w^{-1}(x ; h) ; h\right) .
$$

Therefore, (13) can also be written as

$$
\dot{X}^{W}-\dot{w}\left(w^{-1}\left(X^{\mathscr{W}}\right)\right) \in T_{X^{W}} \Sigma .
$$

From this it follows that each of the terms $\dot{X}^{W} \cdot N^{\mathscr{W}}$ appearing in (11) may be replaced with $\tilde{w} \cdot N^{\mathscr{W}}=w^{\prime}(\cdot ; 0) \cdot N^{\mathscr{W}}$.

$$
\sin \gamma \int_{\partial \Lambda} \tilde{w} \cdot N^{\mathscr{W}}+\cos \gamma \int_{\mathscr{W}} 2 H^{\mathscr{W}} \tilde{w} \cdot N^{\mathscr{W}}+\int_{\mathscr{W}} 2 H \tilde{w} \cdot N^{\mathscr{W}}=0 .
$$

(We are still suppressing the subscript " $m$ " on all the domains of integration; technically, $\partial \Lambda=\partial W_{s} \cup \partial W_{m}$ and the stationary portion of the first integral vanishes because $\dot{X}^{\mathscr{W}} \cdot N^{\mathrm{W}} \equiv 0$ there.)

Next, we temporarily fix $h_{0}$ and consider a specific motion of the wetted region $\mathcal{W}_{h_{0}}$ as follows. Let $\tilde{\mathcal{W}}=\tilde{\mathcal{W}}_{h}$ be parameterized on $M_{m}$ by

$$
p \mapsto w\left(w^{-1}\left(X^{\mathscr{W}}\left(p ; h_{0}\right) ; h_{0}\right) ; h\right) .
$$

Notice that for $h=h_{0}$ the surface $\tilde{\mathcal{W}}$ is exactly $\mathcal{W}_{h_{0}}$ and for $h>h_{0}$ the surface $\tilde{\mathcal{W}}$ is just a rigid motion of the same wetted region and, therefore, has the same area. Thus, using the general formula for the variation of surface area in this special case, we have

$$
0=\frac{d}{d h} A(\tilde{\mathscr{W}})=-\int_{\tilde{\mathscr{W}}} 2 \tilde{H}^{\mathscr{W}} \tilde{w} \cdot \tilde{N}^{\mathscr{W}}+\int_{\partial \tilde{W}} \tilde{w} \cdot \tilde{v}
$$


where $\tilde{H}^{\mathscr{W}}$ is the mean curvature on $\tilde{\mathcal{W}}, \tilde{N}^{\mathcal{W}}$ is the normal on $\tilde{\mathcal{W}}, \tilde{v}$ is the conormal on $\partial \tilde{W}$, and the vectors $\tilde{w}$ appearing in the integrals are evaluated at

$$
\left(w^{-1}\left(w\left(w^{-1}\left(x ; h_{0}\right) ; h\right) ; h_{0}\right) ; h\right) .
$$

We now change variables in the integrals from the previous page using the map $x \mapsto w\left(w^{-1}(x ; h) ; h_{0}\right)$ which maps $\tilde{\mathcal{W}}_{h}$ back to $\tilde{\mathcal{W}}_{h_{0}}=\mathcal{W}_{h_{0}}$. We then have

$$
\begin{array}{rl}
-\int_{\mathscr{W}_{h_{0}}} 2 H^{\mathscr{W}} D & w\left(w^{-1}(\cdot ; h) ; h_{0}\right) D w^{-1}\left(\cdot ; h_{0}\right) w\left(w^{-1}\left(\cdot ; h_{0}\right) ; h\right) \cdot N^{\mathscr{W}} \\
& +\int_{\partial W_{h_{0}}} D w\left(w^{-1}(\cdot ; h) ; h_{0}\right) D w^{-1}\left(\cdot ; h_{0}\right) w\left(w^{-1}\left(\cdot ; h_{0}\right) ; h\right) \cdot \vec{v}=0 .
\end{array}
$$

Setting $h=h_{0}$ and suppressing all the $h$ 's again so that $w\left(w^{-1}\right)=w^{\prime}\left(w^{-1}(\cdot ; h) ; h\right)$, we get

$$
-\int_{\mathscr{W}} 2 H^{\mathscr{W}} \tilde{w}\left(w^{-1}\right) \cdot N^{\mathscr{W}}+\int_{\partial \mathscr{W}} \tilde{w}\left(w^{-1}\right) \cdot \vec{v}=0 .
$$

Using this identity to replace the middle integral on the left in (14), we find at $h=0$ the condition

$$
\int_{\partial \mathscr{W}} w \cdot\left(\sin \gamma N^{\mathscr{W}}+\cos \gamma \vec{v}\right)+\int_{\mathscr{W}} 2 H \tilde{w} \cdot N^{\mathscr{W}} .
$$

Finally, we note that $\sin \gamma N^{\mathrm{W}}+\cos \gamma \vec{v}=\vec{n}$ and modify the notation to emphasize once again the significance of the movable portion $\Sigma_{m}$ of the solid support surface to obtain the fundamental formula.

Theorem 3.1. Assume $X_{0}$ parameterizes a capillary surface $\Lambda_{0}$ that meets solid support surfaces at a constant contact angle $\gamma$ and defines a liquid mass $M$ as described above. If moreover, $\Lambda_{0}$ provides a minimum among variations compatible with the motion of a portion $\Sigma_{m}$ of the bounding surfaces allowed by the rigid motions $w=w(x ; h)$, then

$$
\int_{\mathscr{W}_{m}} 2 H \dot{w}^{\prime} \cdot N^{\mathscr{W}}+\int_{\partial^{\mathscr{W}} W_{m}} \dot{w} \cdot \vec{n}=0,
$$

where $w=\partial w / \partial h(\cdot ; 0)$. It should be noted in this formula that $\vec{n}$ is the conormal to $\partial \Lambda \supset \partial W_{m}$ and $H$ is the mean curvature of $\Lambda$. The formula holds both when $H$ is constant and when $2 H=g-\lambda$ is prescribed by bulk accelerations.

We now apply this result to a particular physical situation.

\section{The floating ball}

Finn [2005] considers the configuration shown in Figure 3 in which a planar interface $z \equiv 0$ for $\alpha=a \sin \gamma \leq r \leq R$ meets a cylindrical container of radius $R$ at an angle $\pi / 2$ and a sphere held rigidly on the axis of the container at an angle of 


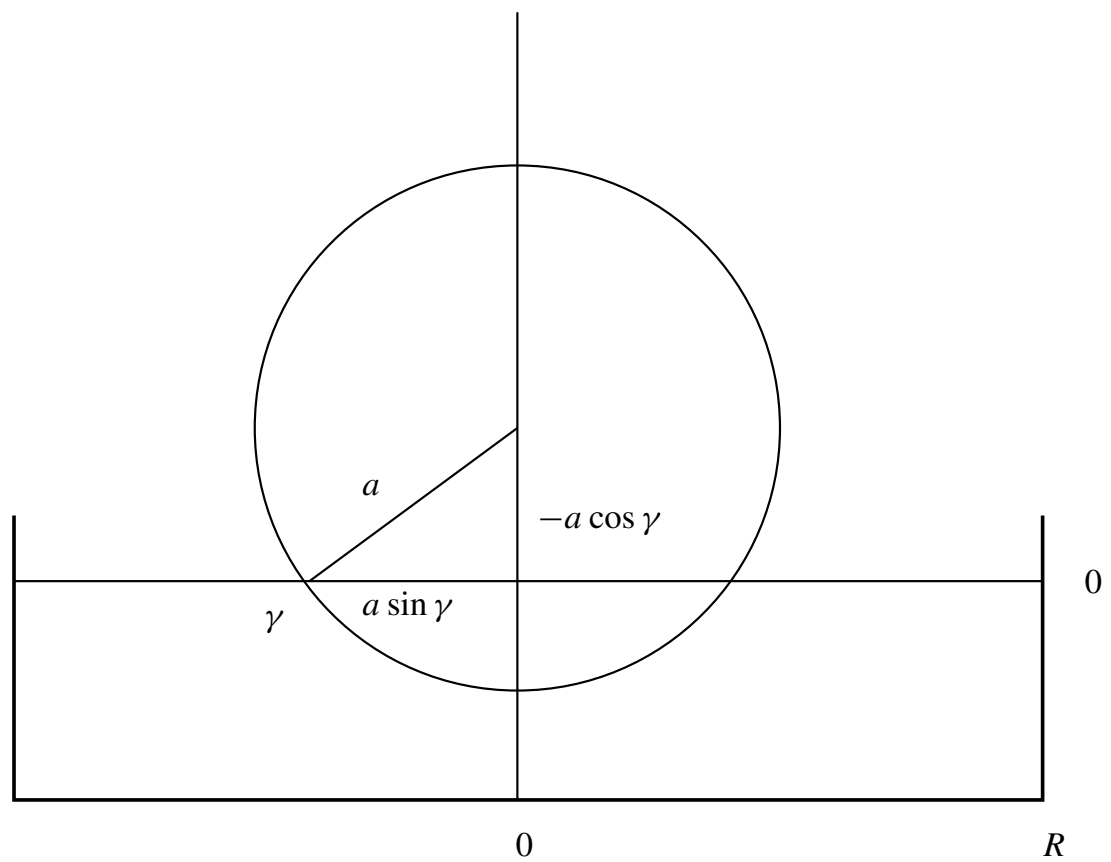

Figure 3. A planar interface determining a liquid mass of given volume and prescribed contact angle.

contact $\gamma$. If the ball remains rigidly fixed with center at height $-a \cos \gamma$, then we may apply the stability theory of Vogel [2000] to obtain the following result whose proof we give at the end of the section.

Theorem 4.1. For the fixed ball problem, the planar interface is linearly stable and is a strong local minimum in the sense of Vogel.

We conclude from this, that such an initial configuration is almost certainly experimentally reproducible, though with some external support to hold the ball in place. One would like to know if the ball will remain in this position and the planar interface will persist if the external support were to be removed. Will the ball float in this position as suggested by Finn?

A theory allowing such a decisive conclusion is apparently unavailable in the situation of moving boundaries. Our calculation leading to Theorem 3.1 however allows us to make an argument that stability with respect to vertical motion of the ball in this problem is also likely.

Finn shows in his paper that if the ball is moved (or suggestively pushed) downward by a small distance $h$, then there is a unique rotationally symmetric constant mean curvature surface that meets the ball still at the angle $\gamma$, the container still at angle $\pi / 2$ and determines a liquid mass of the same volume. The same assertions 


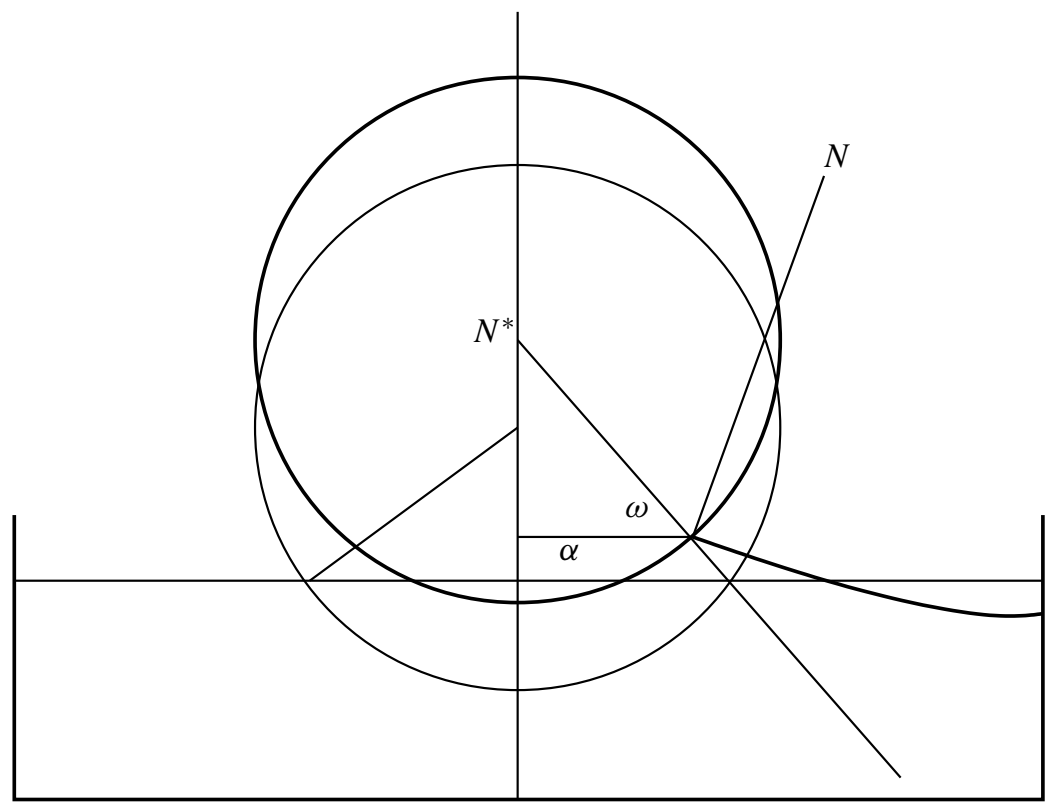

Figure 4. Nodoid interface.

are true if one raises the ball a small distance $h$ (see Figure 4), and the surfaces are, in each case, nodoids whose meridians are obtained as the path of the focal point of a hyperbola as it rolls along the vertical axis through the center of the ball. If for each $h \in(-\delta, \delta)$, these auxiliary configurations have free surface interface $\Lambda_{h}$ and wetted area $\mathscr{W}_{h}$, where the center of the ball is located at $h-a \cos \gamma$, then presumably the overall stability of the configuration (at least with respect to vertical motion of the ball) should be characterized by the values

$$
\mathscr{E}(h)=A\left(\Lambda_{h}\right)-\cos \gamma A\left(W_{h}\right)
$$

We wish to compute, in particular, $\mathscr{\mathscr { E }}=d \mathscr{E} / d h$; the volume term can be ignored, since all the volumes are the same. The quantity $\mathscr{E}(h)$ can be written explicitly in terms of integrals as

$$
\mathscr{E}=2 \pi \int_{\alpha}^{R} r \sqrt{1+u^{\prime}(r)^{2}} d r-\cos \gamma 2 \pi \int_{0}^{\alpha} \frac{a r}{\sqrt{a^{2}-r^{2}}} d r
$$

where $u$ and $\alpha$ are determined as follows. The function $u$ solves the ordinary differential equation of constant mean curvature for rotationally symmetric surfaces. 
This equation is

$$
\frac{1}{r}\left(\frac{r u^{\prime}}{\sqrt{1+u^{\prime 2}}}\right)^{\prime}=2 H_{h}
$$

Denoting by $\alpha=\alpha(h)$ the radius at which the nodoid surface meets the ball, we can integrate to find

$$
u(r)=u(\alpha)+\int_{\alpha}^{r} \frac{\alpha q+H_{h}\left(t^{2}-\alpha^{2}\right)}{\left|H_{h}\right| \sqrt{\left(\rho_{\text {out }}^{2}-t^{2}\right)\left(t^{2}-\rho_{\text {in }}^{2}\right)}} d t,
$$

where $u(\alpha)=h-a \cos \gamma-\sqrt{a^{2}-\alpha^{2}}$, and

$$
q=q(h)=\frac{u^{\prime}(\alpha)}{\sqrt{1+u^{\prime}(\alpha)^{2}}}=-\left(\frac{\alpha}{a} \cos \gamma+\sqrt{1-\left(\frac{\alpha}{a}\right)^{2}} \sin \gamma\right)=-\cos (\gamma-\omega)
$$

where $\omega$ is the smaller angle made by a segment from the center of the ball to the contact line with the horizontal (see Figure 4),

$$
H_{h}=-\frac{\alpha q}{R^{2}-\alpha^{2}}=\frac{\alpha \cos (\gamma-\omega)}{R^{2}-\alpha^{2}}
$$

which follows from the fact that $u^{\prime}(R)=0$ and the first integral of equation (15), and $0<\rho_{\text {in }}<\rho_{\text {out }}$ are the positive roots of $r^{2}-\left[\alpha q+H_{h}\left(r^{2}-\alpha^{2}\right)\right]^{2}=0$. Finally, it remains to describe $\alpha=\alpha(h)$ which is determined implicitly by the equation

$$
\begin{aligned}
& V=\pi R^{2} l+2 \pi \int_{0}^{\alpha} r\left(h-a \cos \gamma-\sqrt{a^{2}-r^{2}}\right) d r+2 \pi \int_{\alpha}^{R} r u(r) d r \\
& =\pi R^{2}(l+h-a \cos \gamma)-2 \pi \int_{0}^{\alpha} r \sqrt{a^{2}-r^{2}} d r \\
& \quad-2 \pi \sqrt{a^{2}-\alpha^{2}} \int_{\alpha}^{R} r d r+2 \pi \int_{\alpha}^{R} r \int_{\alpha}^{r} u^{\prime}(t) d t d r,
\end{aligned}
$$

where $l$ denotes the initial depth of the liquid near the wall of the cylindrical container. In order to show that $\alpha=\alpha(h)$ is well defined for $h$ in some neighborhood of zero, we think of the expression above as defining $V=V(\alpha, h)$ and show that

$$
\frac{\partial V}{\partial \alpha}(a \sin \gamma, 0) \neq 0
$$

This calculation is contained in the proof of Lemma 4.3 below.

The expression for $u$ may also be expressed in terms of standard elliptic integrals, as indicated in [Finn 2005]. Then it will be noted that the volume above as well as $\mathscr{E}$ will depend on integrals of complicated expressions involving elliptic integrals. This should suffice to indicate the difficulty in calculating $\mathscr{E}$ directly. Since we know that $\dot{V}=0$ for this variation, however, we have direct recourse 
to the formula of Theorem 3.1 which is completely independent of the value of $u=u(r)$ for $\alpha<r<R$.

Lemma 4.2. For the floating ball problem described above

$$
\mathscr{\mathscr { E }}=\frac{d \mathscr{E}}{d h}=\frac{2 \pi \alpha R^{2}}{R^{2}-\alpha^{2}} \cos (\gamma-\omega) .
$$

Proof. In this case, the rigid motion is simply translation in the vertical direction $w(x ; h)=x+h \mathbf{e}_{3}$ and $w=\mathbf{e}_{3}$. The wetted region (on the ball) $\mathscr{W}$ is the portion of lower hemisphere $\left\{\left(x, y, h-a \cos \gamma-\sqrt{a^{2}-r^{2}}\right): 0 \leq r^{2}=x^{2}+y^{2} \leq \alpha^{2}\right\}$, and can be parameterized in spherical coordinates $(\theta, \phi)$ for $0 \leq \theta \leq 2 \pi$ and $0 \leq \phi \leq \pi / 2-\omega$. Thus, from the formula in Theorem 3.1,

$$
\begin{aligned}
\mathscr{E}_{\mathscr{E}} & =2 \pi \alpha \mathbf{e}_{3} \cdot\left(\sin \gamma N^{\mathscr{W}}+\cos \gamma \vec{v}\right)+2 H_{h} 2 \pi \int_{0}^{\pi / 2-\omega}\left(\mathbf{e}_{3} \cdot N^{\mathscr{W}}\right) a^{2} \sin \phi d \phi \\
& =2 \pi \alpha(\sin \gamma \sin \omega+\cos \gamma \cos \omega)+\frac{2 \alpha \cos (\gamma-\omega)}{R^{2}-\alpha^{2}} 2 \pi a^{2} \int_{0}^{\pi / 2-\omega} \cos \phi \sin \phi d \phi \\
& =2 \pi \alpha \cos (\gamma-\omega)\left(1+\frac{a^{2}}{R^{2}-\alpha^{2}} \sin ^{2}(\pi / 2-\omega)\right) .
\end{aligned}
$$

Since $a^{2} \sin ^{2}(\pi / 2-\omega)=\alpha^{2}$, this is the formula given above.

Notice that $\alpha$ and $R^{2}-\alpha^{2}$ are both positive. Thus, the sign of $\mathscr{E}$ in this case is determined by the factor $\cos (\gamma-\omega)$. We know that when $h=0$ we have $\omega=\gamma-\pi / 2$ so that $E(0)=0$. In order to determine the value of $\mathscr{E}$ for other values of $h$ we must examine more closely the volume equation for $\alpha=a \cos \omega$.

Lemma 4.3. The function $\alpha=\alpha(h)$ defined in a neighborhood of $h=0$ satisfies $\alpha^{\prime}(0)<0$. Consequently, given $h$ in some neighborhood of zero, since $\cos \omega=\alpha / a$, if $h<0$, then $\omega<\gamma-\pi / 2$; if $h>0$, then $\omega>\gamma-\pi / 2$.

Proof. In this proof, we consider the expressions in the definition of the nodoid meridians (16) to be functions of both $\alpha$ and $h$ as independent variables. From this point of view, we note that the expressions for $q, H$ and $u^{\prime}$ have no explicit dependence on $h$. Furthermore, setting

$$
v=v(\alpha ; r)=\alpha q+H\left(r^{2}-\alpha^{2}\right),
$$

we obtain an alternative expression for $u^{\prime}=u^{\prime}(r ; \alpha)$ :

$$
u^{\prime}=\frac{v}{\sqrt{r^{2}-v^{2}}} \text {. }
$$

Differentiating the expression for the fixed volume (17) with respect to $h$, we find $0=\pi R^{2}+\left(\pi\left(\frac{\alpha}{\sqrt{a^{2}-\alpha^{2}}}-u^{\prime}(\alpha)\right)\left(R^{2}-\alpha^{2}\right)+2 \pi \int_{\alpha}^{R} r \int_{\alpha}^{r} \frac{\partial u^{\prime}}{\partial \alpha}(t) d t d r\right) \alpha^{\prime}(h)$. 
Thus, we wish to show that

$$
\lim _{h \rightarrow 0}\left(\frac{\alpha\left(R^{2}-\alpha^{2}\right)}{\sqrt{a^{2}-\alpha^{2}}}-u^{\prime}(\alpha)\left(R^{2}-\alpha^{2}\right)+2 \int_{\alpha}^{R} r \int_{\alpha}^{r} \frac{\partial u^{\prime}}{\partial \alpha}(t) d t d r\right)>0 .
$$

We consider each term individually; each term has a completely nonsingular limit. First,

$$
\frac{\alpha\left(R^{2}-\alpha^{2}\right)}{\sqrt{a^{2}-\alpha^{2}}} \longrightarrow-\tan \gamma\left(R^{2}-a^{2} \sin ^{2} \gamma\right)>0 \quad \text { as } h \rightarrow 0,
$$

where we have used that $-\tan \gamma>0$. Since $q$ and $H$ tend to 0 with $h$, we see that $v$ tends uniformly to zero for $r$ in some neighborhood of $[a \sin \gamma, R]$. In particular, $\alpha u^{\prime}(\alpha)$ tends to 0 with $h$, so the second term vanishes in the limit.

The last term requires some preliminary calculations and limits:

$$
\frac{\partial u^{\prime}}{\partial \alpha}=\frac{r^{2}}{\left(r^{2}-v^{2}\right)^{3 / 2}} \frac{\partial v}{\partial \alpha}=\frac{r^{2}}{\left(r^{2}-v^{2}\right)^{3 / 2}}\left(q+\alpha \frac{\partial q}{\partial \alpha}+\frac{\partial H}{\partial \alpha}\left(r^{2}-\alpha^{2}\right)-2 \alpha H\right) .
$$

Inside the parentheses we have, as $h \rightarrow 0$,

$$
\frac{\partial q}{\partial \alpha}=-\frac{1}{a} \cos \gamma+\frac{\alpha}{a \sqrt{a^{2}-\alpha^{2}}} \sin \gamma \longrightarrow-\frac{1}{a}(\cos \gamma+\tan \gamma \sin \gamma)=-\frac{1}{a \cos \gamma}
$$

and

$$
\frac{\partial H}{\partial \alpha}=-\frac{\partial}{\partial \alpha}\left(\frac{\alpha}{R^{2}-\alpha^{2}}\right) q-\left(\frac{\alpha}{R^{2}-\alpha^{2}}\right) \frac{\partial q}{\partial \alpha} \longrightarrow \frac{\tan \gamma}{R^{2}-a^{2} \sin ^{2} \gamma}
$$

Therefore,

$$
\frac{\partial u^{\prime}}{\partial \alpha} \longrightarrow \frac{1}{r}\left(-\tan \gamma+\tan \gamma \frac{r^{2}-a^{2} \sin ^{2} \gamma}{R^{2}-a^{2} \sin ^{2} \gamma}\right)=-\frac{\tan \gamma}{r\left(R^{2}-a^{2} \sin ^{2} \gamma\right)}\left(R^{2}-r^{2}\right)
$$

as $h \rightarrow 0$. Since $-\tan \gamma>0$, this shows the last term in (18) also has a positive limit.

We recall that each of the nodoid interfaces is considered a critical interface for the fixed ball problem. That is, they satisfy the necessary conditions of having constant mean curvature and constant contact angle equal to $\gamma$ on the contact line with the ball. With the addition of our third necessary condition in the floating ball problem, we can now state the following.

Theorem 4.4. With respect to the variation of the planar interface through nodoid interfaces parameterized by the height of the ball $h$, the plane is the only equilibrium for $h$ in some neighborhood of $h=0$ and provides a strict minimum of energy among those interfaces. 
We were unable to analyze precisely the nodoid interfaces (even concerning existence and connectedness) for $h$ far from zero, but since the formula in Lemma 4.2 results only from boundary considerations and we can say that $\cos (\gamma-\omega) \neq 0$ for every nodoid interface, we can make the following assertion:

Theorem 4.5. The unique rotationally symmetric equilibrium for the floating ball problem is the planar interface; each nodoid interface is unstable to first order with respect to motion of the ball.

It is, of course, unlikely that any of the other nodoid interfaces would have lower energy than the plane. Presumably, an analytic argument could be given to cover the component of those interfaces connected via $h$ to the neighborhood of the planar interface considered in Theorem 4.4, and the global picture could be understood numerically if not analytically. For now, we have illustrated the use of Theorem 3.1.

Lastly, we return to the fixed ball problem and show that the planar interface is a strong local minimum in the sense of Vogel.

Proof of Theorem 4.1. This configuration satisfies the conditions stated in [Vogel 2000]. Therefore, the stability for the interface is determined by the eigenvalue problem

$$
\begin{cases}\Delta \phi+\lambda \phi=0, & r=\sqrt{x^{2}+y^{2}} \in(\alpha, R), \\ \left.\frac{\partial \phi}{\partial r}\right|_{r=\alpha}=\left.\frac{1}{\alpha} \phi\right|_{r=\alpha} ; & \left.\frac{\partial \phi}{\partial r}\right|_{r=R} \equiv 0 .\end{cases}
$$

Vogel shows that if all eigenvalues for this problem are positive, the interface is strictly stable and a strong local minimum with respect to a large class of perturbations.

In polar coordinates, the equation becomes

$$
\frac{1}{r}\left(\frac{\partial}{\partial r}\left(r \frac{\partial \phi}{\partial r}\right)+\frac{\partial}{\partial \theta}\left(\frac{1}{r} \frac{\partial \phi}{\partial \theta}\right)\right)+\lambda \phi=0 .
$$

Setting

$$
\phi=\sum_{k=1}^{\infty} g_{k}(r) \sin (k \theta)+\sum_{k=0}^{\infty} f_{k}(r) \cos (k \theta),
$$

we are led to the Sturm-Liouville problems

$$
\left\{\begin{array}{l}
r f^{\prime \prime}+f^{\prime}+\left(\lambda r-k^{2} / r\right) f=0, \quad \alpha<r<R, \\
f^{\prime}(\alpha)=\frac{1}{\alpha} f(\alpha) ; \quad f^{\prime}(R)=0,
\end{array}\right.
$$

where $f$ denotes $f_{k}, k=0,1, \ldots$ or $g_{k}, k=1,2, \ldots$ 
We wish to show that there are no nontrivial solutions for $\lambda \leq 0$. For $\lambda<0$ we set $\lambda=-\mu^{2}$ and change variables $(t=\mu r)$ to obtain

$$
\left\{\begin{array}{l}
f^{\prime \prime}+(1 / t) f^{\prime}-\left(1+k^{2} / t^{2}\right) f=0, \quad \alpha \mu<t<R \mu \\
f^{\prime}(\mu \alpha)=\frac{1}{\mu \alpha} f(\mu \alpha) ; \quad f^{\prime}(\mu R)=0 .
\end{array}\right.
$$

This is a regular problem which we can transform using the change of variables $\dot{\tau}=\log t$. Then we get

$$
f(t)=v(\log t) ; \quad f^{\prime}=\frac{v^{\prime}}{t}, \quad f^{\prime \prime}=\frac{v^{\prime \prime}}{t^{2}}-\frac{v^{\prime}}{t^{2}},
$$

so that

$$
\left\{\begin{array}{l}
v^{\prime \prime}-\left(e^{2 \tau}+k^{2}\right) v=0, \quad l_{1}=\log (\mu \alpha)<\tau<\log (\mu R)=l_{2} \\
v^{\prime}\left(l_{1}\right)=v\left(l_{1}\right) ; \quad v^{\prime}\left(l_{2}\right)=0
\end{array}\right.
$$

We claim that this problem has no nontrivial solution. In fact, if $v\left(l_{1}\right)<0$, then $v^{\prime}\left(l_{1}\right)=v\left(l_{1}\right)<0$ and $v^{\prime \prime}\left(l_{1}\right)=\left(e^{2 l_{1}}+k^{2}\right) v\left(l_{1}\right)<0$. Since each of $v(\tau), v^{\prime}(\tau)$, and $v^{\prime \prime}(\tau)$ must remain negative, there can be no $l_{2}>l_{1}$ for which $v^{\prime}\left(l_{2}\right)=0$. (Assume that one of $v, v^{\prime}, v^{\prime \prime}$ vanishes first at $\tau=\tau_{1}$. If $v\left(\tau_{1}\right)=0$, there is some $\tau_{2}<\tau_{1}$ for which $v^{\prime}\left(\tau_{2}\right)>0$, a contradiction. The same argument holds if $v^{\prime}\left(\tau_{1}\right)=0$. If $v^{\prime \prime}\left(\tau_{1}\right)=0$, then

$$
v\left(\tau_{1}\right)=\frac{1}{\left(e^{2 l_{1}}+k^{2}\right)} v^{\prime}\left(\tau_{2}\right)=0,
$$

also a contradiction.)

If $v\left(l_{1}\right)=0$, then uniqueness of solutions gives $v \equiv 0$.

If $v\left(l_{1}\right)>0$, then $v, v^{\prime}$, and $v^{\prime \prime}$ must remain forever positive, so we cannot have $v^{\prime}\left(l_{2}\right)=0$.

If $\lambda=0$, the problem becomes

$$
\left\{\begin{array}{l}
r^{2} f^{\prime \prime}+r f^{\prime}-k^{2} f=0, \\
f^{\prime}(\alpha)=\frac{1}{\alpha} f(\alpha) ; \quad f^{\prime}(R)=0 .
\end{array}\right.
$$

This is an Euler equation that transforms as above to

$$
\begin{cases}v^{\prime \prime}=k^{2} v, & l_{1}=\log \alpha<\tau<\log R=l_{2}, \\ v^{\prime}\left(l_{1}\right)=v\left(l_{1}\right) ; & v^{\prime}\left(l_{2}\right)=0 .\end{cases}
$$


An argument similar to that in the case $\lambda<0$, shows that only the trivial solution is possible. In fact, more explicitly we have $v=c e^{k t}+d e^{-k t}$, so that

$$
\left\{\begin{array}{l}
c e^{k l_{1}}-d e^{-k l_{1}}=c e^{k l_{1}}+d e^{-k l_{1}}, \\
c e^{k l_{2}}-d e^{-k l_{2}}=0
\end{array}\right.
$$

or

$$
\left\{\begin{array}{l}
(k-1) e^{k l_{1}} c-(k+1) e^{-k l_{1}} d=0, \\
e^{k l_{2}} c-e^{-k l_{2}} d=0,
\end{array}\right.
$$

which has only the trivial solution since

$$
\begin{aligned}
-(k-1) e^{k\left(l_{1}-l_{2}\right)}+(k+1) e^{k\left(l_{2}-l_{1}\right)} & =k\left(e^{k\left(l_{2}-l_{1}\right)}-e^{-k\left(l_{2}-l_{1}\right)}\right)+e^{k\left(l_{2}-l_{1}\right)}+e^{-k\left(l_{2}-l-1\right)} \\
& =2 k \sinh k\left(l_{2}-l_{1}\right)+2 \cosh k\left(l_{2}-l_{1}\right) \neq 0 .
\end{aligned}
$$

(If this were to vanish, we would have

$$
\frac{\sinh k\left(l_{2}-l_{1}\right)}{\cosh k\left(l_{2}-l_{1}\right)}=-\frac{1}{k}<0, \quad k \neq 0
$$

or

$$
\cosh k\left(l_{2}-l_{1}\right)=0, \quad k=0,
$$

both of which are impossible.)

Thus, the interface under consideration is stable and also a strong local minimum by the work of Vogel.

\section{References}

[Barbosa and do Carmo 1984] J. L. Barbosa and M. do Carmo, "Stability of hypersurfaces with constant mean curvature”, Math. Z. 185:3 (1984), 339-353. MR 85k:58021c Zbl 0513.53002

[Barbosa et al. 1988] J. L. Barbosa, M. do Carmo, and J. Eschenburg, "Stability of hypersurfaces of constant mean curvature in Riemannian manifolds", Math. Z. 197:1 (1988), 123-138. MR 88m:53109 Zbl 0653.53045

[Blaschke 1930] W. Blaschke, Vorlesungen über Differentialgeometrie und geometrische Grundlagen von Einsteins Relativitätstheorie, I: Elementare Differentialgeometrie, 3rd ed., Grundlehren der Math. Wiss. 1, Springer, Berlin, 1930. Reprinted Dover, New York, 1945. JFM 56.0588.07

[Ekeland and Temam 1976] I. Ekeland and R. Temam, Convex analysis and variational problems, Studies in Mathematics and its Applications 1, North-Holland, Amsterdam, 1976. MR 57 \#3931b Zbl 0322.90046

[Evans and Gariepy 1992] L. C. Evans and R. F. Gariepy, Measure theory and fine properties of functions, CRC Press, Boca Raton, FL, 1992. MR 93f:28001 Zbl 0804.28001

[Finn 1986] R. Finn, Equilibrium capillary surfaces, Grundlehren der Math. Wissenschaften 284, Springer, New York, 1986. MR 88f:49001 Zbl 0583.35002

[Finn 2005] R. Finn, "Floating and partly immersed balls in a weightless environment", Funct. Differ. Equ. 12:1-2 (2005), 167-173. MR 2005m:76072 Zbl 1085.76016 
[Gelfand and Fomin 1963] I. M. Gelfand and S. V. Fomin, Calculus of variations, Prentice-Hall, Englewood Cliffs, NJ, 1963. Reprinted Dover, New York, 2000. MR 28 \#3353 Zbl 0127.05402

[McCuan 2006] J. McCuan, "Variation of energy for capillary surfaces", Preprint, MPI-MIS, Leipzig, 2006, Available at http://www.mis.mpg.de/preprints/2006/prepr2006_18.html.

[Ros and Souam 1997] A. Ros and R. Souam, "On stability of capillary surfaces in a ball”, Pacific J. Math. 178:2 (1997), 345-361. MR 98c:58029 Zbl 0930.53007

[Sagan 1969] H. Sagan, Introduction to the calculus of variations, McGraw-Hill, New York, 1969. Reprinted Dover, New York, 1992. MR 94h:49001

[Serrin 1959] J. Serrin, "Mathematical principles of classical fluid mechanics", pp. 125-263 in Handbuch der Physik, 8/1: Strömungsmechanik I, edited by S. Flügge and C. Truesdell, Springer, Berlin, 1959. MR 21 \#6836b

[Spivak 1979] M. Spivak, A comprehensive introduction to differential geometry, vol. 4, 2nd ed., Publish or Perish, Wilmington, DE, 1979. MR 82g:53003d Zbl 0439.53004

[Vogel 2000] T. I. Vogel, "Sufficient conditions for capillary surfaces to be energy minima", Pacific J. Math. 194:2 (2000), 469-489. MR 2001j:58023 Zbl 1021.58014

[Wente 1966] H. C. Wente, Existence theorems for surfaces of constant mean curvature and perturbations of a liquid globule in equilibrium, Ph.D. thesis, Harvard University, Cambridge, MA, 1966.

Received January 11, 2006. Revised June 9, 2006.

\section{JOHN MCCUAN}

SCHOOL OF MATHEMATICS

GeORgIA INSTITUTE OF TECHNOLOGY

686 CHERRY STREET

ATLANTA, GA 30332

UNITED STATES

mccuan@math.gatech.edu

http://www.math.gatech.edu/ mccuan 